\title{
The effects of plant-based diets on the body and the brain: a systematic review
}

\author{
Evelyn Medawar (10 ${ }^{1,2,3}$, Sebastian Huhn ${ }^{4}$, Arno Villringer ${ }^{1,2,3}$ and A. Veronica Witte
}

\begin{abstract}
Western societies notice an increasing interest in plant-based eating patterns such as vegetarian and vegan, yet potential effects on the body and brain are a matter of debate. Therefore, we systematically reviewed existing human interventional studies on putative effects of a plant-based diet on the metabolism and cognition, and what is known about the underlying mechanisms. Using the search terms "plant-based OR vegan OR vegetarian AND diet AND intervention" in PubMed filtered for clinical trials in humans retrieved 205 studies out of which 27, plus an additional search extending the selection to another five studies, were eligible for inclusion based on three independent ratings. We found robust evidence for short- to moderate-term beneficial effects of plant-based diets versus conventional diets (duration $\leq 24$ months) on weight status, energy metabolism and systemic inflammation in healthy participants, obese and type-2 diabetes patients. Initial experimental studies proposed novel microbiome-related pathways, by which plant-based diets modulate the gut microbiome towards a favorable diversity of bacteria species, yet a functional "bottom up" signaling of plant-based diet-induced microbial changes remains highly speculative. In addition, little is known, based on interventional studies about cognitive effects linked to plant-based diets. Thus, a causal impact of plant-based diets on cognitive functions, mental and neurological health and respective underlying mechanisms has yet to be demonstrated. In sum, the increasing interest for plant-based diets raises the opportunity for developing novel preventive and therapeutic strategies against obesity, eating disorders and related comorbidities. Still, putative effects of plant-based diets on brain health and cognitive functions as well as the underlying mechanisms remain largely unexplored and new studies need to address these questions.
\end{abstract}

\section{Introduction \\ Background}

Western societies notice an increasing interest in plantbased eating patterns such as avoiding meat or fish or fully excluding animal products (vegetarian or vegan, see Fig. 1). In 2015, around 0.4-3.4\% US adults, $1-2 \%$ British adults, and $5-10 \%$ of German adults were reported to eat largely plant-based $\operatorname{diets}^{1-4}$, due to various reasons (reviewed in ref. ${ }^{5}$ ). Likewise, the number of scientific publications on PubMed (Fig. 2) and the public popularity as depicted by Google Trends (Fig. 3) underscore the

\footnotetext{
Correspondence: Evelyn Medawar (medawar@cbs.mpg.de)

'Department of Neurology, Max Planck Institute for Human Cognitive and Brain Sciences, Leipzig, Germany

${ }^{2}$ Berlin School of Mind and Brain, Humboldt-Universität zu Berlin, Berlin, Germany

Full list of author information is available at the end of the article.
}

increased interest in plant-based diets. This increasing awareness calls for a better scientific understanding of how plant-based diets affect human health, in particular with regard to potentially relevant effects on mental health and cognitive functions.

\section{Study aims}

A potential effect of plant-based diets on mortality rate remains controversial: large epidemiological studies like the Adventist studies $(n=22,000-96,000)$ show a link between plant-based diets, lower all-cause mortality and cardiovascular diseases ${ }^{6,7}$, while other studies like the EPIC-Oxford study and the " 45 and Up Study" ( $n=$ $64,000-267,000)$ show none ${ }^{8,9}$. Yet, many, but not all, epidemiological and interventional human studies in the last decades have suggested that plant-based diets exert beneficial health effects with regard to obesity-related

\section{(c) The Author(s) 2019}

(c) (i) Open Access This article is licensed under a Creative Commons Attribution 4.0 International License, which permits use, sharing, adaptation, distribution and reproduction in any medium or format, as long as you give appropriate credit to the original author(s) and the source, provide a link to the Creative Commons license, and indicate if changes were made. The images or other third party material in this article are included in the article's Creative Commons license, unless indicated otherwise in a credit line to the material. If material is not included in the article's Creative Commons license and your intended use is not permitted by statutory regulation or exceeds the permitted use, you will need to obtain permission directly from the copyright holder. To view a copy of this license, visit http://creativecommons.org/licenses/by/4.0/. 
metabolic dysfunction, type 2 diabetes mellitus (T2DM) and chronic low-grade inflammation (e.g. refs. ${ }^{6,7,10,11}$, for reviews, see refs. ${ }^{12-18}$ ). However, while a putative link between such metabolic alterations and brain health through pathways which might include diet-related neurotransmitter precursors, inflammatory pathways and the gut microbiome ${ }^{19}$ becomes increasingly recognized, the notion that plant-based diets exert influence on mental health and cognitive functions appears less documented and controversial ${ }^{20-24}$. We therefore systematically reviewed the current evidence based on available

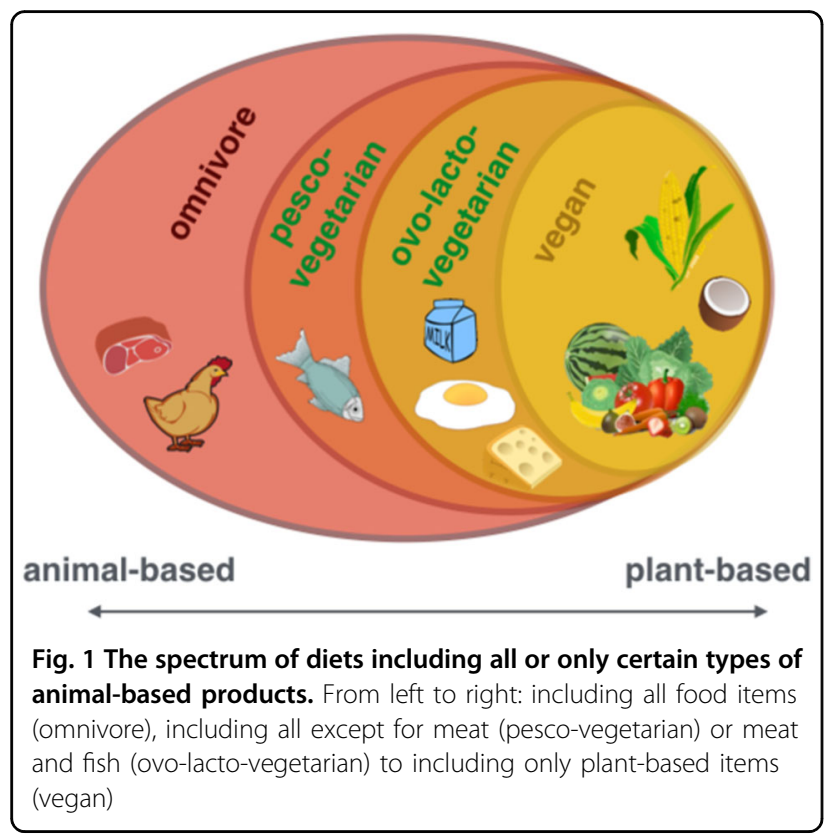

controlled interventional trials, regarded as the gold standard to assess causality, on potential effects of plantbased diets on (a) metabolic factors including the microbiome and (b) neurological or psychiatric health and brain functions. In addition, we aimed to evaluate potential underlying mechanisms and related implications for cognition.

\section{Methods}

We performed a systematic PubMed search with the following search terms "plant-based OR vegan $O R$ vegetarian AND diet AND intervention" with the filter "clinical trial" and "humans", preregistered at PROSPERO (CRD42018111856; https://www.crd.york.ac. uk/PROSPERO/display_record.php?RecordID $=111856$ )

(Suppl. Fig. 1). PubMed was used as search engine because it was esteemed to yield the majority of relevant human clinical trials from a medical perspective. Exclusion criteria were insufficient design quality (such as lack of a control group), interventions without a plant-based or vegetarian or vegan diet condition, intervention with multiple factors (such as exercise and diet), and the exclusive report of main outcomes of no interest, such as dietary compliance, nutrient intake (such as vitamins or fiber intake), or nonmetabolic (i.e., not concerning glucose metabolism, lipid profile, gastrointestinal hormones or inflammatory markers) or non-neurological/psychiatric disease outcomes (e.g. cancer, caries).

Studies were independently rated for eligibility into the systematic review by three authors based on reading the abstract and, if needed, methods or other parts of the publication. If opinions differed, a consensus was reached

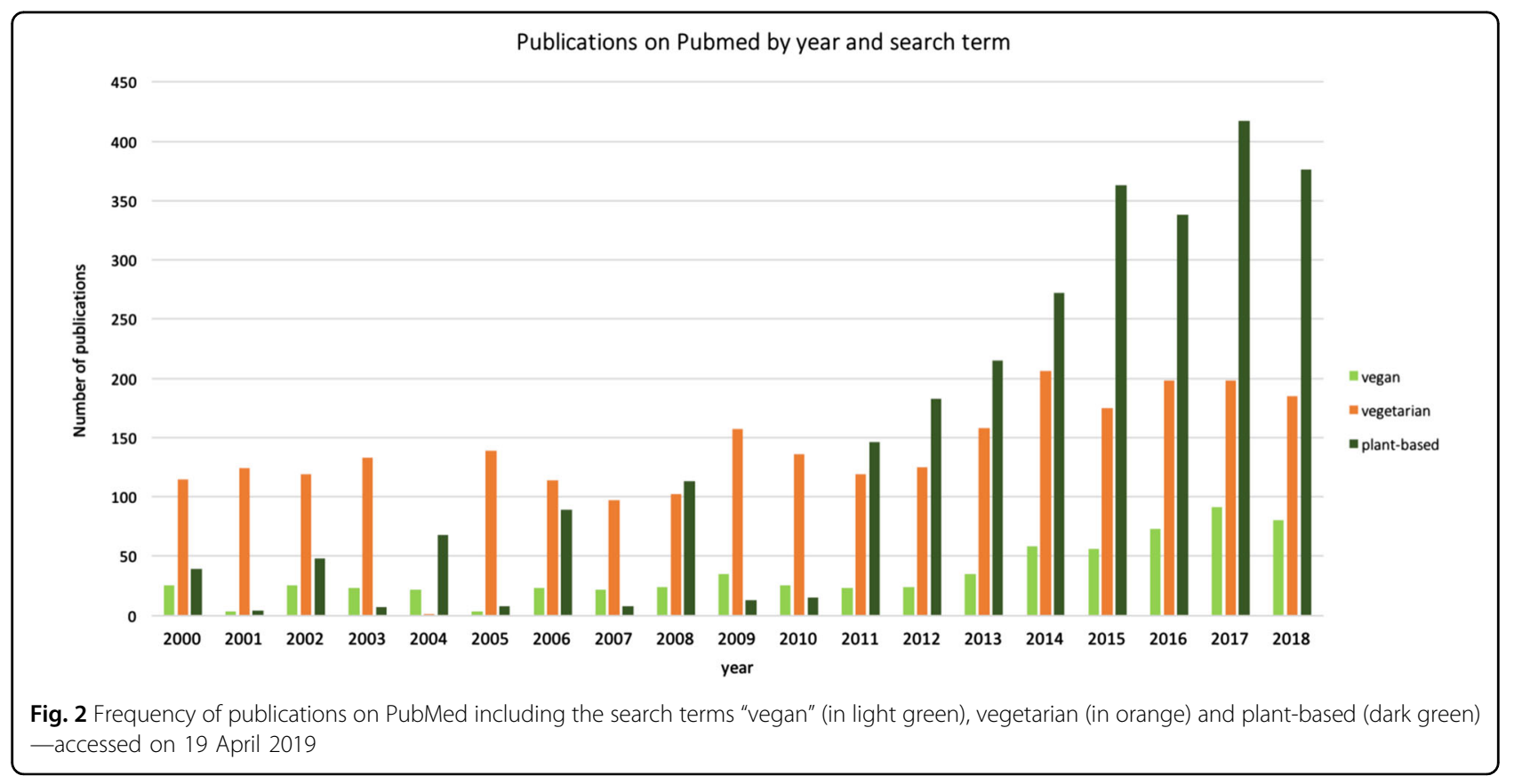



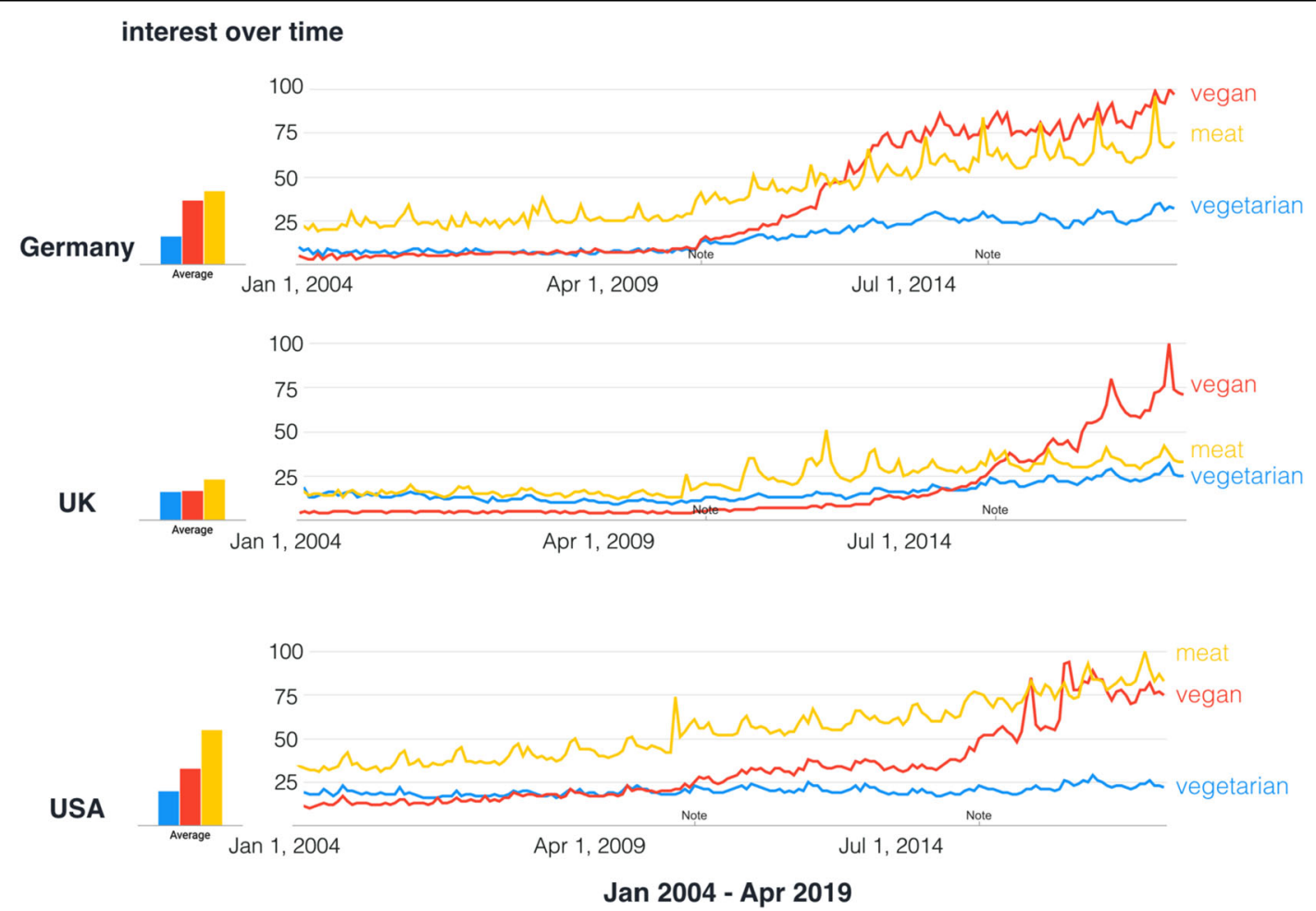

Fig. 3 Google Trends Search for search term hits for "vegan", "vegetarian" and "meat" in Germany (adapted to "vegetarisch", "vegan" and "fleisch"), the USA and the UK from 2004 to present. Note indicates technical improvements implemented by Google Trends. Data source: Google Trends. Search performed on 18 April 2019

through discussion of the individual study. This yielded 27 eligible out of 205 publications; see Table 1 for details. To increase the search radius for studies dealing with microbial and neurological/psychiatric outcomes, we deleted the search term "intervention", which increased the number of studies by around one third, and checked for studies with "microbiome/microbiota", "mental", "cognitive/cognition" or "psychological/psychology" in the resulting records. Through this, we retrieved another five studies included in Table 1. Further related studies were reviewed based on additional nonsystematic literature search.

\section{Section I: Effects of plant-based diets on body and brain outcomes}

Results based on interventional studies on metabolism, microbiota and brain function

Overall, the vast majority of studies included in this systematic review reported a short-term beneficial effect of plant-based dietary interventions (study duration 3 -24 months) on weight status, glucose, insulin and/or plasma lipids and inflammatory markers, whereas studies investigating whether plant-based diets affect microbial or neurological/psychiatric disease status and other brain functions were scarce and rather inconclusive (Table 1).
More specifically, 19 out of 32 studies dealing with T2DM and/or obese subjects and seven out of 32 dealing with healthy subjects observed a more pronounced weight loss and metabolic improvements, such as lowering of glycated hemoglobin (HbA1c) - a long-term marker for glucose levels-decreased serum levels of low-density (LDL) and high-density lipoproteins (HDL) and total cholesterol (TC), after a plant-based diet compared to an omnivore diet. This is largely in line with recent metaanalyses indicating beneficial metabolic changes after a plant-based diet ${ }^{25-27}$.

For example, Lee et al. found a significantly larger reduction of HbA1c and lower waist circumference after vegan compared to conventional dieting ${ }^{28}$. Jenkins et al. found a disease-attenuating effect in hyperlipidemic patients after 6 months adopting a low-carbohydrate plant-based diet compared to a high-carbohydrate lactoovo-vegetarian $\operatorname{diet}^{29,30}$. However, lower energy intake in the vegan dieters might have contributed to these effects. Yet, while a plant-based diet per se might lead to lower caloric intake, other studies observed nonsignificant trends toward higher effect sizes on metabolic parameters after a vegan diet, even when caloric intake was comparable: two studies in T2DM patients ${ }^{31,32}$ compared calorie-unrestricted vegan or vegetarian to calorie- 


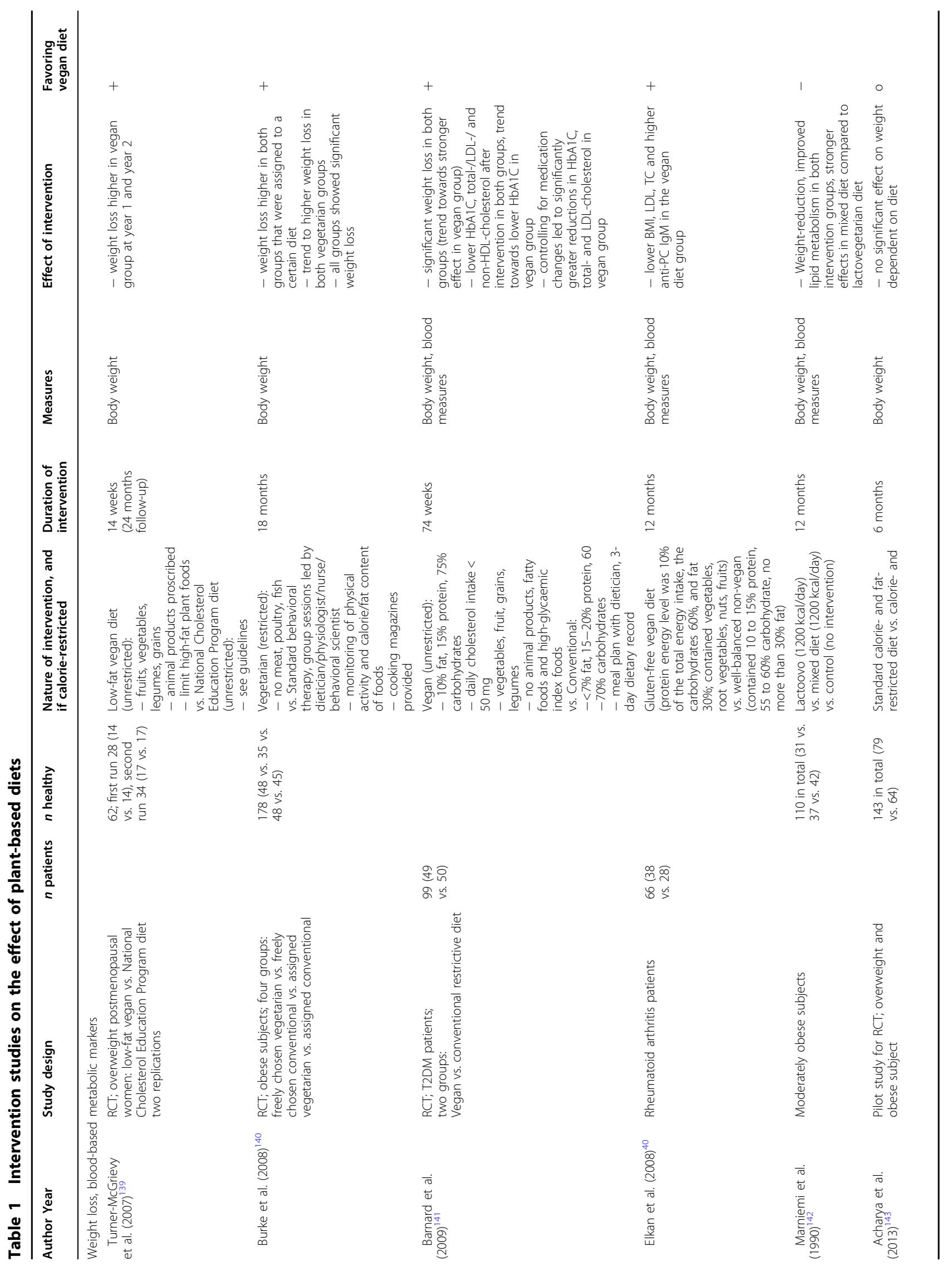




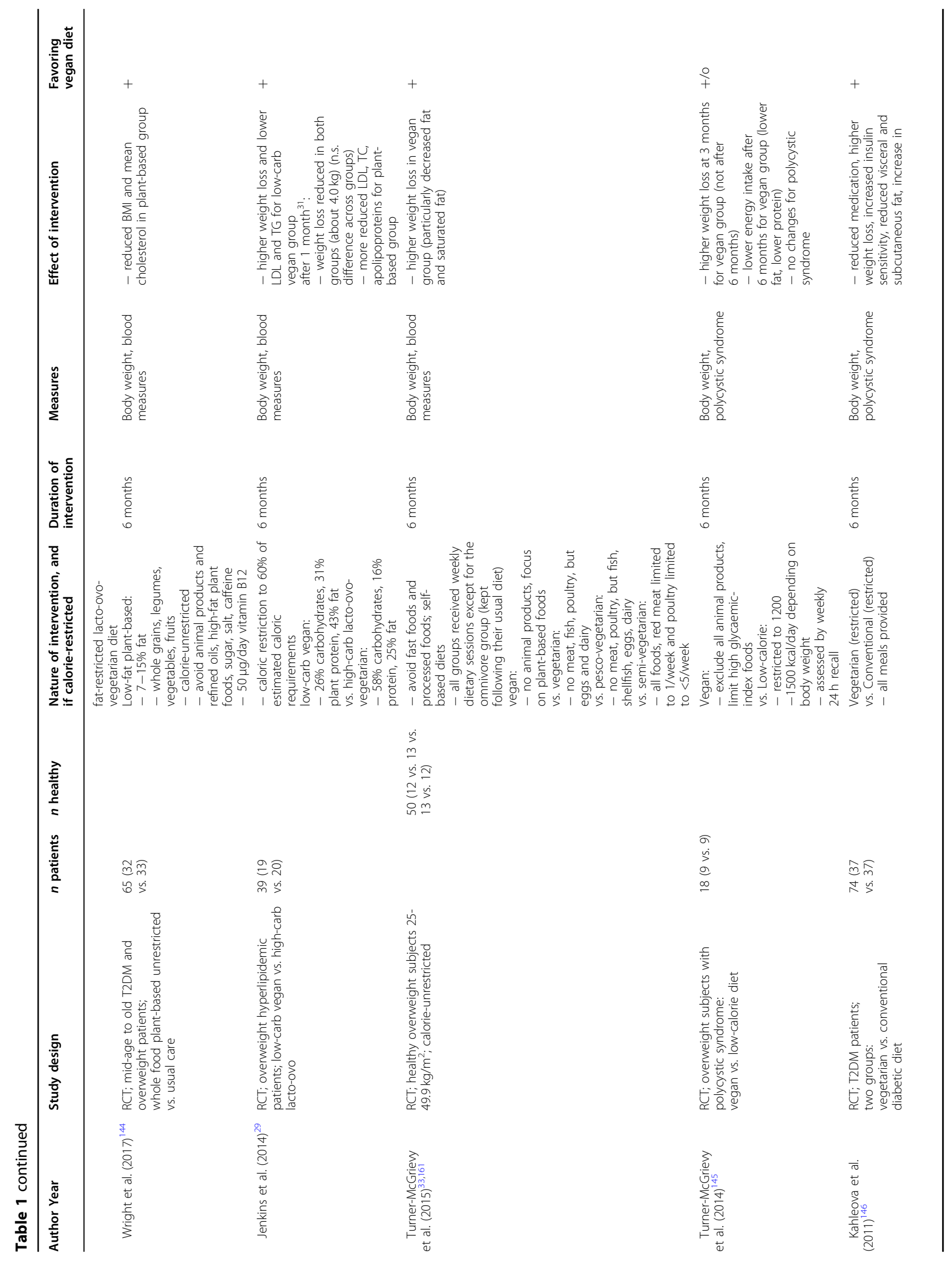




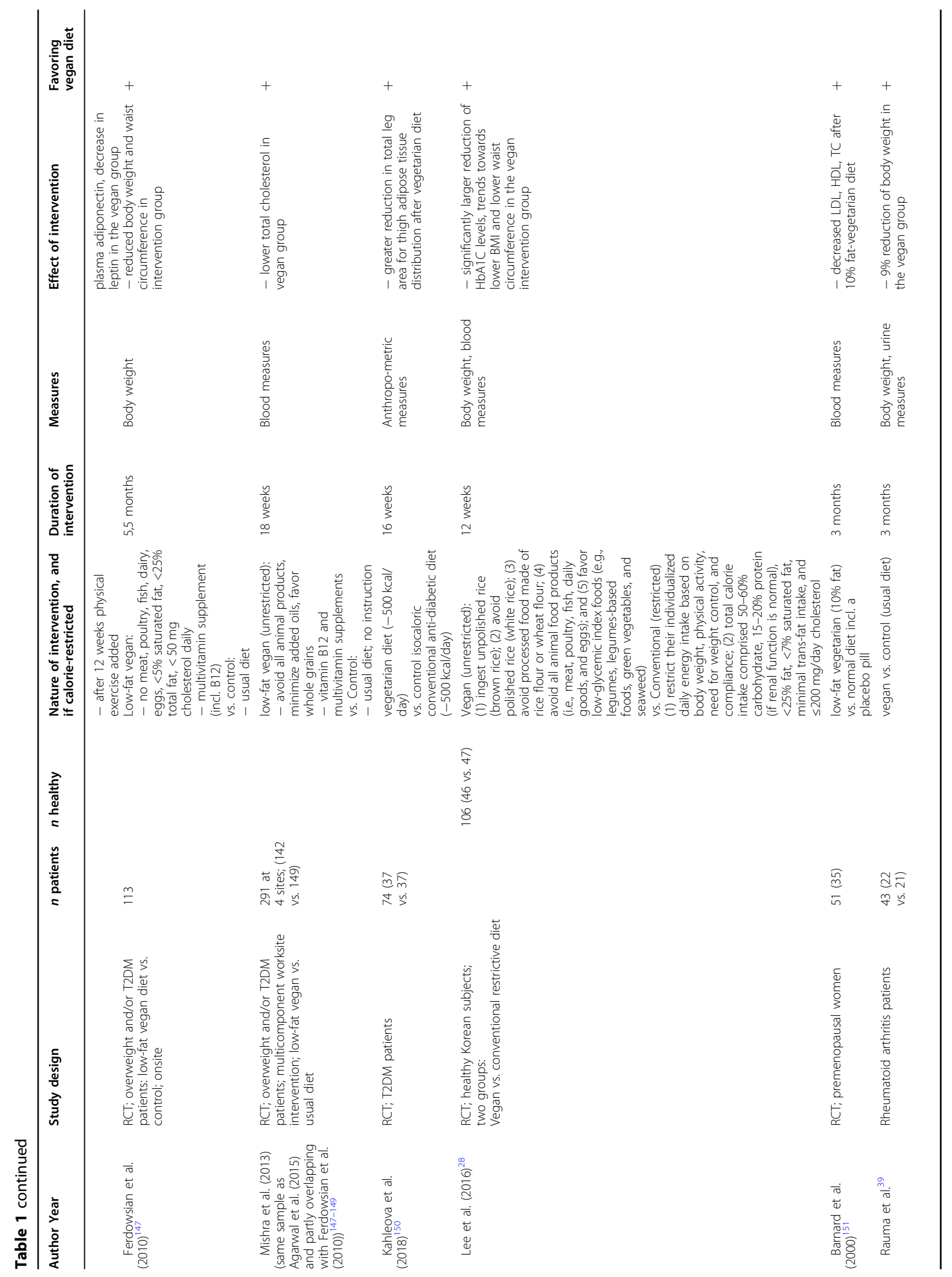




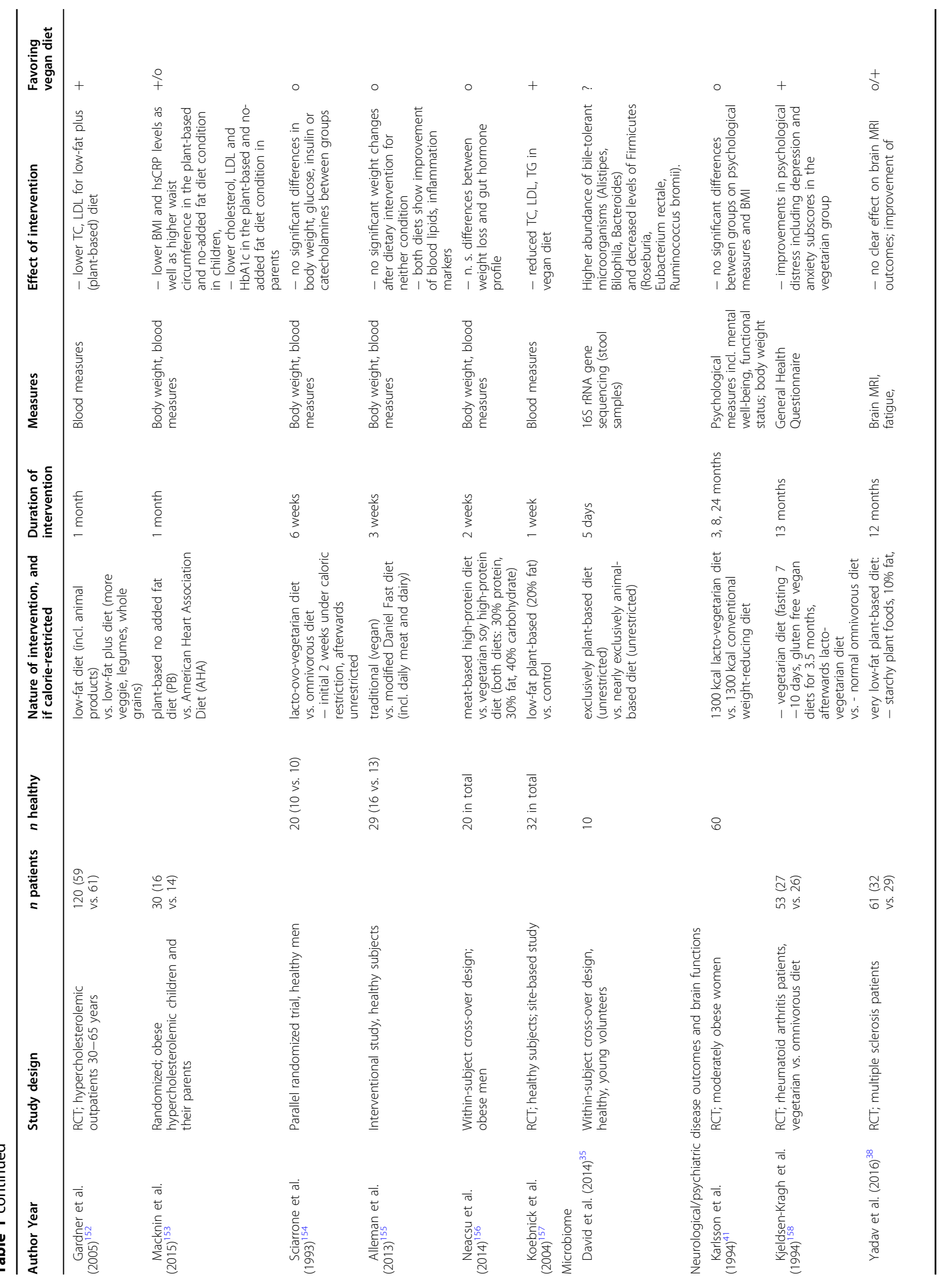




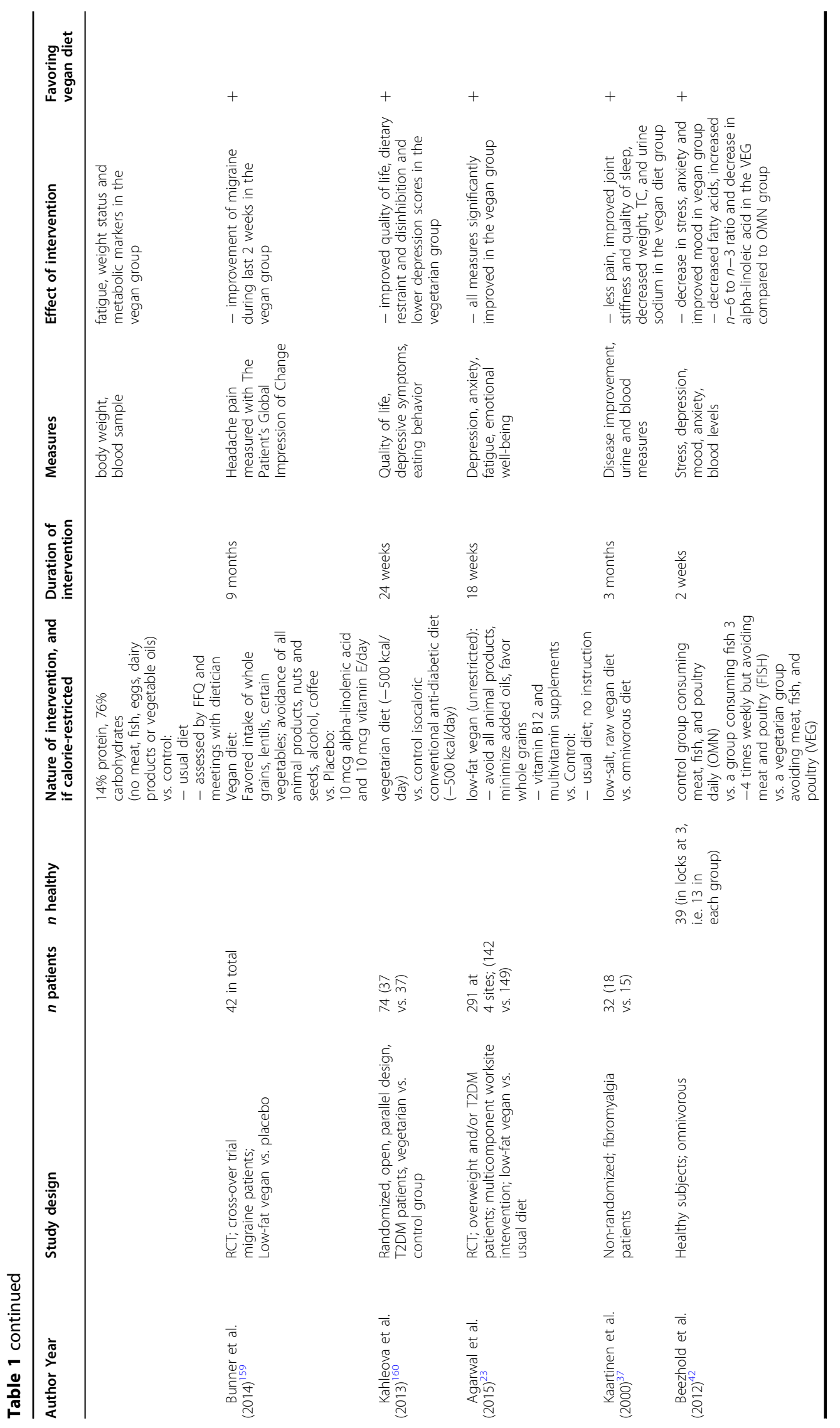


restricted conventional diets over periods of 6 months and 1.5 years, respectively, in moderate sample sizes $(n \sim 75$ -99) with similar caloric intake achieved in both diet groups. Both studies indicated stronger effects of plantbased diets on disease status, such as reduced medication, improved weight status and increased glucose/insulin sensitivity, proposing a diabetes-preventive potential of plant-based diets. Further, a five-arm study comparing four types of plant-based diets (vegan, vegetarian, pescovegetarian, semi-vegetarian) to an omnivore diet (total $n=63$ ) in obese participants found the most pronounced effect on weight loss for a vegan diet $(-7.5 \pm 4.5 \%$ of total body weight $)^{33}$. Here, inflammation markers conceptualized as the dietary inflammatory index were also found to be lower in vegan, vegetarian and pescovegetarian compared to semi-vegetarian overweight to obese dieters ${ }^{33}$.

Intriguingly, these results ${ }^{28-33}$ cohesively suggest that although caloric intake was similar across groups, participants who had followed a vegan diet showed higher weight loss and improved metabolic status.

As a limitation, all of the reviewed intervention studies were carried out in moderate sample sizes and over a period of less than 2 years, disregarding that long-term success of dietary interventions stabilizes after 2-5 years only $^{34}$. Future studies with larger sample sizes and tight control of dietary intake need to confirm these results.

Through our systematic review we retrieved only one study that added the gut microbiome as novel outcome for clinical trials investigating the effects of animal-based diets compared to plant-based diets. While the sample size was relatively low $(n=10$, cross-over within subject design), it showed that changing animal- to plant based diet changed gut microbial activity towards a trade-off between carbohydrate and protein fermentation processes within only 5 days $^{35}$. This is in line with another controlled-feeding study where microbial composition changes already occurred $24 \mathrm{~h}$ after changing diet (not exclusively plant-based) ${ }^{36}$. However, future studies incorporating larger sample sizes and a uniform analysis approach of microbial features need to further confirm the hypothesis that a plant-based diet ameliorates microbial diversity and health-related bacteria species.

Considering neurological or psychiatric diseases and brain functions, the systematic review yielded in six clinical trials of diverse clinical groups, i.e. migraine, multiple sclerosis, fibromyalgia and rheumatoid arthritis. Here, mild to moderate improvement, e.g. measured by antibody levels, symptom improvement or pain frequency, was reported in five out of six studies, sometimes accompanied by weight $\operatorname{loss}^{37-40}$ (Table 1). However, given the pilot character of these studies, indicated by small sample sizes $(n=32-66)$, lack of randomization ${ }^{37}$, or that the plant-based diet was additionally free of gluten ${ }^{40}$, the evidence is largely anecdotal. One study in moderately obese women showed no effects on psychological outcomes ${ }^{41}$, two studies with obese and nonobese healthy adults indicated improvements in anxiety, stress and depressive symptom scores ${ }^{23,24}$. Taken together, the current evidence based on interventional trials regarding improvements of cognitive and emotional markers and in disease treatment for central nervous system disorders such as multiple sclerosis or fibromyalgia remains considerably fragmentary for plant-based diets.

Among observational studies, a recent large crosssectional study showed a higher occurrence of depressive symptoms for vegetarian dieters compared to nonvegetarians ${ }^{20}$. Conversely, another observational study with a sample of about $80 \%$ women found a beneficial association between a vegan diet and mood disturbance ${ }^{24}$.

Overall, the relationship between mental health (i.e. depression) and restrictive eating patterns has been the focus of recent research ${ }^{20-22,24,42}$; however, causal relationships remain uninvestigated due to the observational design.

\section{Underlying mechanisms linking macronutrient intake to metabolic processes}

On the one hand, nutrient sources as well as their intake ratios considerably differ between plant-based and omnivore diets (Suppl. Table 1), and on the other hand, dietary micro- and macromolecules as well as their metabolic substrates affect a diversity of physiological functions, pointing to complex interdependencies. Thus, it seems difficult to nail down the proposed beneficial effects of a plant-based diet on metabolic status to one specific component or characteristic, and it seems unlikely that the usually low amount of calories in plant-based diets could explain all observed effects. Rather, plantbased diets might act through multiple pathways, including better glycemic control ${ }^{43}$, lower inflammatory activity $^{44}$ and altered neurotransmitter metabolism via dietary intake ${ }^{45}$ or intestinal activity ${ }^{46}$ (Fig. 4).

On the macronutrient level, plant-based diets feature different types of fatty acids (mono- and poly-unsaturated versus saturated and trans) and sugars (complex and unrefined versus simple and refined), which might both be important players for mediating beneficial health effects ${ }^{18}$. On the micronutrient level, the EPIC-Oxford study provided the largest sample of vegan dieters worldwide $(n$ $($ vegan $)=2396, n($ total $)=65,429)$ and showed on the one hand lower intake of saturated fatty acids (SFA), retinol, vitamin $\mathrm{B} 12$ and $\mathrm{D}$, calcium, zinc and protein, and on the other hand higher intake of fiber, magnesium, iron, folic acid, vitamin $\mathrm{B} 1, \mathrm{C}$ and $\mathrm{E}$ in vegan compared to omnivore dieters ${ }^{47}$. Other studies confirmed the variance of nutrient intake across dietary groups, i.e. omnivores, vegetarians and vegans, showing the occurrence of critical nutrients 


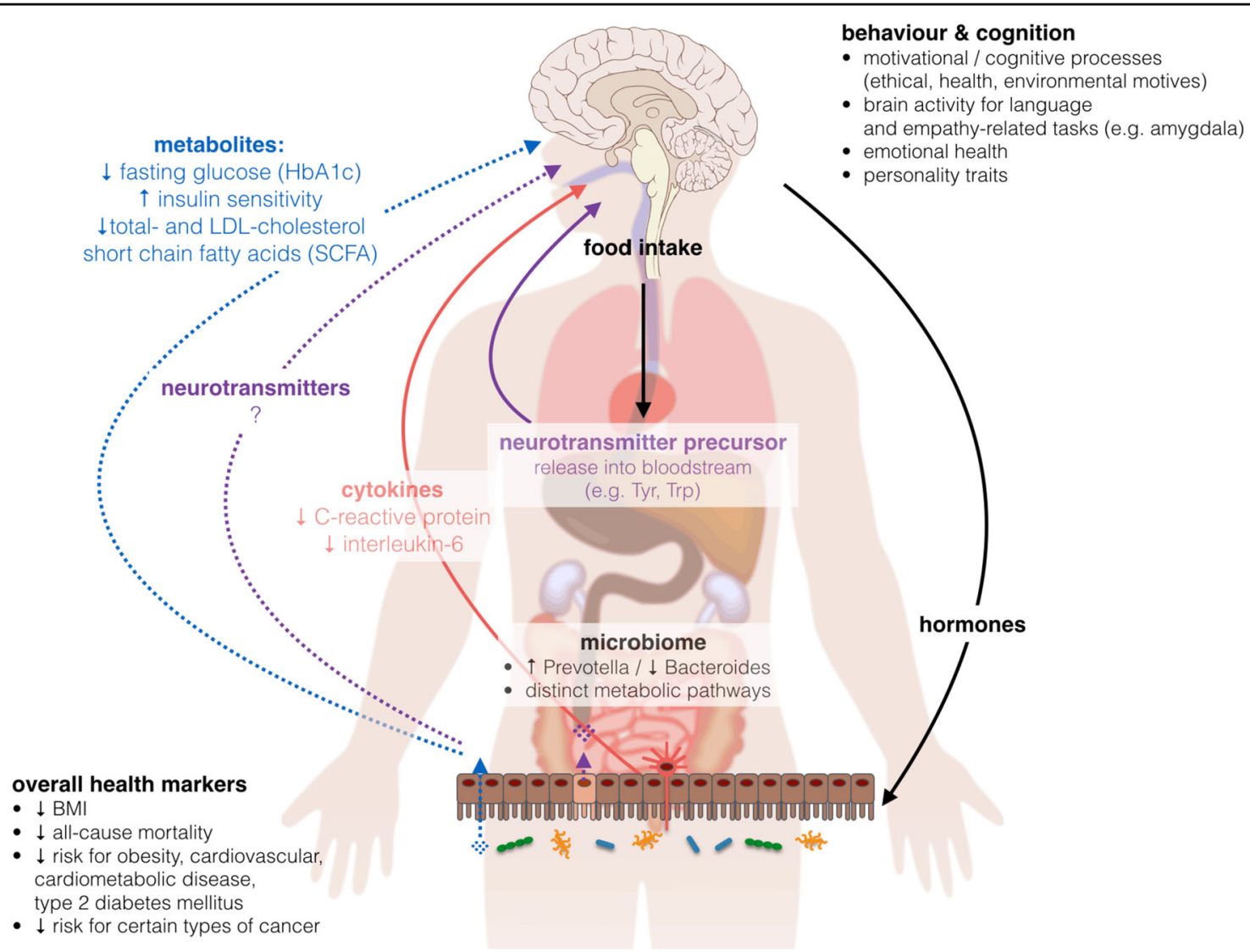

Fig. 4 The effects of a plant-based diet on the microbiome-gut-brain axis including the here reviewed effects on overall health, microbial composition and activity, behavior and cognition. BMI body-mass-index, HbA1c hemoglobin A1c, LDL-cholesterol low-density lipoprotein cholesterol, Trp tryptophan, Tyr tyrosine. Images from commons.wikimedia.org, "Brain human sagittal section" by Lynch 2006 and "Complete Gl tract" by Häggström 2008, "Anatomy Figure Vector Clipart" by http://moziru.com

for each group ${ }^{48,49}$. Not only the amount of SFA but also its source and profile might be important factors regulating metabolic control (reviewed in ref. ${ }^{14}$ ), for example through contributing to systemic hyperlipidemia and subsequent cardiovascular risk. Recently, it has been shown in a 4-week intervention trial that short-term dietary changes favoring a diet high in animal-based protein may lead to an increased risk for cardiovascular derangements mediated by higher levels of trimethylamine $\mathrm{N}$-oxide (TMAO), which is a metabolite of gut bacteria-driven metabolic pathways ${ }^{50}$.

Secondly, high fiber intake from legumes, grains, vegetables and fruits is a prominent feature of plant-based diets (Table 1), which could induce beneficial metabolic processes like upregulated carbohydrate fermentation and downregulated protein fermentation ${ }^{35}$, improved gut hormonal-driven appetite regulation ${ }^{51-55}$, and might prevent chronic diseases such as obesity and T2DM by slowing down digestion and improving lipid control ${ }^{56}$. A comprehensive review including evidence from 185 prospective studies and 58 clinical trials concluded that risk reduction for a myriad of diseases (incl. CVD, T2DM, stroke incidence) was greatest for daily fiber intake between 25 and $29 \mathrm{~g}^{57}$. Precise evidence for underlying mechanisms is missing; however, more recently it has been suggested that high fiber intake induces changes on the microbial level leading to lower long-term weight gain $^{58}$, a mechanism discussed below.

The reason for lower systemic inflammation in plantbased dieters could be due to the abundance of antiinflammatory molecule intake and/or avoidance of proinflammatory animal-derived molecules. Assessing systemic inflammation is particularly relevant for medical conditions such as obesity, where it has been proposed to increase the risk for cardiovascular disease ${ }^{59,60}$. In addition, higher C-reactive protein (CRP) and interleukin-6 (IL-6) levels have been linked with measures of brain microstructure, such as microstructural integrity and white matter lesions ${ }^{61-63}$ and higher risk of dementia ${ }^{64}$, and recent studies point out that a diet-related low inflammatory index might also directly affect healthy brain ageing ${ }^{65,66}$. 
Interventional studies that focus on plant- versus meatbased proteins or micronutrients and potential effects on the body and brain are lacking. A meta-analysis including seven RCTs and one cross-sectional studies on physical performance and dietary habits concluded that a vegetarian diet did not adversely influence physical performance compared to an omnivore $\operatorname{diet}^{67}$. An epidemiological study by Song et al. ${ }^{11}$ estimated that statistically replacing 3\% of animal protein, especially from red meat or eggs, with plant protein would significantly improve mortality rates. This beneficial effect might however not be explained by the protein source itself, but possibly by detrimental components found in meat (e.g. heme-iron or nitrosamines, antibiotics, see below).

Some studies further hypothesized that health benefits observed in a plant-based diet stem from higher levels of fruits and vegetables providing phytochemicals or vitamin $\mathrm{C}$ that might boost immune function and eventually prevent certain types of cancer ${ }^{68-70}$. A meta-analysis on the effect of phytochemical intake concluded a beneficial effect on CVD, cancer, overweight, body composition, glucose tolerance, digestion and mental health ${ }^{71}$. Looking further on the impact of micronutrients and single dietary compounds, there is room for speculation that molecules, that are commonly avoided in plant-based diets, might affect metabolic status and overall health, such as opioidpeptides derived from casein $^{72}$, pre- and probiotics ${ }^{73,74}$, carry-over antibiotics found in animal products ${ }^{75,76}$ or food-related carcinogenic toxins, such as dioxin found in eggs or nitrosamines found in red and processed meat ${ }^{77,78}$. Although conclusive evidence is missing, these findings propose indirect beneficial effects on health deriving from plant-based compared to animal-based foods, with a potential role for nonprotein substances in mediating those effects ${ }^{18}$. While data regarding chemical contaminant levels (such as crop pesticides, herbicides or heavy metals) in different food items are fragmentary only, certain potentially harmful compounds may be more (or less) frequently consumed in plant-based diets compared to more animal-based diets ${ }^{79}$. Whether these differences lead to systematic health effects need to be explored.

Taken together, the reviewed studies indicating effects of plant-based diets through macro- and micronutrient intake reveal both the potential of single ingredients or food groups (low SFA, high fiber) and the immense complexity of diet-related mechanisms for metabolic health. As proposed by several authors, benefits on health related to diet can probably not be viewed in isolation for the intake (or nonintake) of specific foods, but rather by additive or even synergistic effects between them (reviewed in refs. ${ }^{12,80}$ ). Even if it remains a challenging task to design long-term RCTs that control macro- and micronutrient levels across dietary intervention groups, technological advancements such as more fine-tuned diagnostic measurements and automated self-monitoring tools, e.g. automatic food recognition systems ${ }^{81}$ and urine-related measures of dietary intake ${ }^{82}$, could help to push the field forward.

\section{Nutrients of particular interest in plant-based diets}

As described above, plant-based diets have been shown to convey nutritional benefits ${ }^{48,49}$, in particular increased fiber, beta carotene, vitamin $\mathrm{K}$ and $\mathrm{C}$, folate, magnesium, and potassium intake and an improved dietary health index ${ }^{83}$. However, a major criticism of plant-based diets is the risk of nutrient deficiencies for specific micronutrients, especially vitamin B12, a mainly animal-derived nutrient, which is missing entirely in vegan diets unless supplemented or provided in B12-fortified products, and which seems detrimental for neurological and cognitive health when intake is low. In the EPIC-Oxford study about $50 \%$ of the vegan dieters showed serum levels indicating vitamin B12 deficiency ${ }^{84}$. Along other risk factors such as age ${ }^{85}$, diet, and plant-based diets in particular, seem to be the main risk factor for vitamin B12 deficiency (reviewed in ref. ${ }^{86}$ ), and therefore supplementing vitamin B12 for these risk groups is highly recommended $^{87}$. Vitamin B12 is a crucial component involved in early brain development, in maintaining normal central nervous system function ${ }^{88}$ and suggested to be neuroprotective, particularly for memory performance and hippocampal microstructure ${ }^{89}$. One hypothesis is that high levels of homocysteine, that is associated with vitamin B12 deficiency, might be harmful to the body. Vita$\min \mathrm{B} 12$ is the essential cofactor required for the conversion of homocysteine into nonharmful components and serves as a cofactor in different enzymatic reactions. A person suffering from vitamin B12 insufficiency accumulates homocysteine, lastly promoting the formation of plaques in arteries and thereby increasing atherothrombotic risk ${ }^{90}$, possibly facilitating symptoms in patients of Alzheimer's disease ${ }^{91}$. A meta-analysis found that vitamin B12 deficiency was associated with stroke, Alzheimer's disease, vascular dementia, Parkinson's disease and in even lower concentrations with cognitive impairment ${ }^{92}$, supporting the claim of its high potential for disease prevention when avoided or treated ${ }^{93}$. Further investigations and longitudinal studies are needed, possibly measuring holotranscobalamin (the active form of vitamin B12) as a more specific and sensitive marker for vitamin B12 status $^{94}$, to examine in how far nonsupplementing vegan dieters could be at risk for cardiovascular and cognitive impairment.

Similar health dangers can stem from iron deficiency, another commonly assumed risk for plant-based dieters and other risk groups such as young women. A meta- 
analysis on 24 studies proposes that although serum ferritin levels were lower in vegetarians on average, it is recommended to sustain an optimal ferritin level (neither too low nor too high), calling for well-monitored supplementation strategies ${ }^{95}$. Iron deficiency is not only dependent on iron intake as such but also on complimentary dietary factors influencing its bioavailability (discussed in ref. ${ }^{95}$ ). The picture remains complex: on the one hand iron deficiency may lead to detrimental health effects, such as impairments in early brain development and cognitive functions in adults and in children carried by iron-deficient mothers ${ }^{96}$ and a possible role for iron overload in the brain on cognitive impairment on the other hand ${ }^{97}$. One study showed that attention, memory and learning were impaired in iron-deficient compared to iron-sufficient women, which could be restored after a 4month oral iron supplementation $(n=118)^{98}$. Iron deficiency-related impairments could be attributed to anemia as an underlying cause, possibly leading to fatigue, or an undersupply of blood to the brain or alterations in neurobiological and neuronal systems $^{99}$ provoking impaired cognitive functioning.

This leads to the general recommendation to monitor health status by frequent blood tests, to consult a dietician to live healthily on a plant-based diet and to consider supplements to avoid nutrient deficiencies or nutrientoverdose-related toxicity. All in all, organizations such as the Academy of Nutrition and Dietetics ${ }^{100}$ and the German Nutrition Society do not judge iron as a major risk factor for plant-based dieters ${ }^{101}$.

\section{Section II: Effects of diet on the gut microbiome The link between diet and microbial diversity}

Another putative mechanistic pathway of how plantbased diets can affect health may involve the gut microbiome which has increasingly received scientific and popular interest, lastly not only through initiatives such as the Human Microbiome Project ${ }^{102}$. A common measure for characterizing the gut community is enterotyping, which is a way to stratify individuals according to their gut bacterial diversity, by calculating the ratio between bacterial genera, such as Prevotella and Bacteroides ${ }^{103}$. While interventional controlled trials are still scarce, this ratio has been shown to be conclusive for differentiating plantbased from animal-based microbial profiles ${ }^{36}$. Specifically, in a sample of 98 individuals, Wu et al. ${ }^{36}$ found that a diet high in protein and animal fats was related to more Bacteroides, whereas a diet high in carbohydrates, representing a plant-based one, was associated with more Prevotella. Moreover, the authors showed that a change in diet to high-fat/low-fiber or to low-fat/high-fiber in ten individuals elicited a change in gut microbial enterotype with a time delay of $24 \mathrm{~h}$ only and remained stable over 10 days, however not being able to switch completely to another enterotype ${ }^{36}$. Another strictly controlled 30-day cross-over interventional study showed that a change in diet to either an exclusively animal-based or plant-based diet promoted gut microbiota diversity and genetic expression to change within 5 days $^{35}$. Particularly, in response to adopting an animal-based diet, microbial diversity increased rapidly, even overshadowing individual microbial gene expression. Beyond large shifts in overall diet, already modest dietary modifications such as the daily consumption of $43 \mathrm{~g}$ of walnuts, were able to promote probiotic- and butyric acid-producing bacterial species in two RCTs, after 3 and 8 weeks respectively ${ }^{104,105}$, highlighting the high adaptability of the gut microbiome to dietary components. The Prevotella to Bacteroides ratio $(\mathrm{P} / \mathrm{B})$ has been shown to be involved in the success of dietary interventions targeting weight loss, with larger weight loss in high $\mathrm{P} / \mathrm{B}$ compared to low $\mathrm{P} / \mathrm{B}$ in a 6-month whole-grain diet compared to a conventional diet ${ }^{106}$. Only recently, other microbial communities, such as the salivary microbiome, have been shown to be different between omnivores and vegan dieters ${ }^{107}$, opening new avenues for research on adaptable mechanisms related to dietary intake.

\section{A continuum in microbial diversity dependent on diet}

Plant-based diets are supposed to be linked to a specific microbial profile, with a vegan profile being most different from an omnivore, but not always different from a vegetarian profile (reviewed in ref. ${ }^{15}$ ). Some specifically vegan gut microbial characteristics have also been found in a small sample of six obese subjects after 1 month following a vegetarian diet, namely less pathobionts, more protective bacterial species improving lipid metabolism and a reduced level of intestinal inflammation ${ }^{108}$. Investigating long-term dietary patterns a study found a dosedependent effect for altered gut microbiota in vegetarians and vegans compared to omnivores depending on the quantity of animal products ${ }^{109}$. The authors showed that gut microbial profiles of plant-based diets feature the same total number but lower counts of Bacteroides, Bifidobacterium, E. coli and Enterobacteriaceae compared to omnivores, with the biggest difference to vegans. Still today it remains unclear, what this shift in bacterial composition means in functional terms, prompting the field to develop more functional analyses.

In a 30-day intervention study, David et al. found that fermentation processes linked to fat and carbohydrate decomposition were related to the abundance of certain microbial species ${ }^{35}$. They found a strong correlation between fiber intake and Prevotella abundance in the microbial gut. More recently, Prevotella has been associated with plant-based diets ${ }^{110}$ that are comparable to low-fat/high-fiber diets ${ }^{111}$ and might be linked to the increased synthesis of short-chain fatty acids (SCFA) ${ }^{112}$. 
SCFAs are discussed as putative signaling molecules between the gut microbiome and the receptors, i.e. free fatty acid receptor 2 (FFA2) ${ }^{51}$, found in host cells across different tissues ${ }^{113}$ and could therefore be one potential mechanism of microbiome-host communication.

The underlying mechanisms of nutrient decomposition by Prevotella and whether abundant Prevotella populations in the gut are beneficial for overall health remain unknown. Yet it seems possible that an increased fiber intake and therefore higher Prevotella abundance such as associated with plant-based diets is beneficial for regulating glycemic control and keeping inflammatory processes within normal levels, possibly due to reduced appetite and lower energy intake mediated by a higher fiber content ${ }^{114}$. Moreover, it has been brought forward that the microbiome might influence bodily homeostatic control, suggesting a role for the gut microbiota in wholebody control mechanisms on the systemic level. Novel strategies aim to develop gut-microbiota-based therapies to improve bodily states, e.g. glycemic control ${ }^{115}$, based on inducing microbial changes and thereby eliciting higherlevel changes in homeostasis. While highly speculative, such strategies could in theory also exert changes on the brain level, which will be discussed next in the light of a bi-directional feedback between the gut and the brain.

\section{Effects on cognition and behavior linking diet and cognition via the microbiome-gut-brain axis}

While the number of interventional studies focusing on cognitive and mental health outcomes after adopting plant-based diets overall is very limited (see Section I above), one underlying mechanism of how plant-based diets may affect mood could involve signaling pathways on the microbiome-gut-brain axis ${ }^{116-119}$. A recent 4week intervention RCT showed that probiotic administration compared to placebo and no intervention modulated brain activity during emotional decision-making and emotional recognition tasks ${ }^{117}$. In chronic depression it has been proposed that immunoglobulin $\mathrm{A}$ and $\mathrm{M}$ antibodies are synthesized by the host in response to gut commensals and are linked to depressive symptoms ${ }^{120}$. Whether the identified gram-negative bacteria might also play a role in plant-based diets remains to be explored. A meta-analysis on five studies concluded that probiotics may mediate an alleviating effect on depression symptomatic $^{121}$-however, sample sizes remained rather small $(n<100)$ and no long-term effects were tested (up to 8 weeks).

Currently, several studies aim to identify microbial profiles in relation to disease and how microbial data can be used on a multimodal way to improve functional resolution, e.g. characterizing microbial profiles of individuals suffering from type- 1 diabetes ${ }^{122}$. Yet, evidence for specific effects of diet on cognitive functions and behavior through changes in the microbiome remains scarce. A recent study indicated the possibility that our food choices determine the quantity and quality of neurotransmitterprecursor levels that we ingest, which in turn might influence behavior, as shown by lower fairness during a money-redistribution task, called the ultimatum game, after a high-carbohydrate/protein ratio breakfast than after a low-ratio breakfast ${ }^{123}$. Strang et al. found that precursor forms of serotonin and dopamine, measured in blood serum, predicted behavior in this task, and precursor concentrations were dependent on the nutrient profile of the consumed meal before the task. Also on a cross-sectional level tryptophan metabolites from fecal samples have been associated with amygdala-reward network functional connectivity ${ }^{124}$. On top of the dietary composition per se, the microbiota largely contributes to neurotransmitter precursor concentrations; thus, in addition to measuring neurotransmitter precursors in the serum, metabolomics on fecal samples would be helpful to further understand the functional role of the gut microbiota in neurotransmitter biosynthesis and regulation $^{125}$.

Indicating the relevance of gut microbiota for cognition, a first human study assessing cognitive tests and brain imaging could distinguish obese from nonobese individuals using a microbial profile ${ }^{126}$. The authors found a specific microbiotic profile, particularly defined by Actinobacteria phylum abundance, that was associated with microstructural properties in the hypothalamus and in the caudate nucleus. Further, a preclinical study tested whether probiotics could enhance cognitive function in healthy subjects, showing small effects on improved memory performance and reduced stress levels ${ }^{127}$.

A recent study could show that microbial composition influences cerebral amyloidogenesis in a mouse model for Alzheimer's disease $^{128}$. Health status of the donor mouse seemingly mattered: fecal transplants from transgenic mice had a larger impact on amyloid beta proliferation in the brain compared to wild-type feces. Translational interpretations to humans should be done with caution if at all-yet the results remain elucidative for showing a link between the gut microbiome and brain metabolism.

The evidence for effects of strictly plant-based diets on cognition is very limited. For other plant-based diets such as the Mediterranean diet or DASH diet, there are more available studies that indicate protective effects on cardiovascular and brain health in the aging population (reviewed in refs. ${ }^{129,130}$ ). Several attempts have been made to clarify potential underlying mechanisms, for example using supplementary plant polyphenols, fish/ fish-oil consumption or whole dietary pattern change in $\mathrm{RCTs}^{131-137}$, yet results are not always equivocal and large-scale intervention studies have yet to be completed. 
The overall findings of this paragraph add to the evidence that microbial diversity may be associated with brain health, although underlying mechanisms and candidate signaling molecules remain unknown.

\section{Conclusion}

Based on this systematic review of randomized clinical trials, there is an overall robust support for beneficial effects of a plant-based diet on metabolic measures in health and disease. However, the evidence for cognitive and mental effects of a plant-based diet is still inconclusive. Also, it is not clear whether putative effects are due to the diet per se, certain nutrients of the diet (or the avoidance of certain animal-based nutrients) or other factors associated with vegetarian/vegan diets. Evolving concepts argue that emotional distress and mental illnesses are linked to the role of microbiota in neurological function and can be potentially treated via microbial intervention strategies ${ }^{19}$. Moreover, it has been claimed that certain diseases, such as obesity, are caused by a specific microbial composition ${ }^{138}$, and that a balanced gut microbiome is related to healthy ageing ${ }^{111}$. In this light, it seems possible that a plant-based diet is able to influence brain function by still unclear underlying mechanisms of an altered microbial status and systemic metabolic alterations. However, to our knowledge there are no studies linking plant-based diets and cognitive abilities on a neural level, which are urgently needed, due to the hidden potential as a dietary therapeutic tool. Also, further studies are needed to disentangle motivational beliefs on a psychological level that lead to a change in diet from causal effects on the body and the brain mediated e.g., by metabolic alterations or a change in the gut microbiome.

\section{Acknowledgements}

This work was supported by a scholarship (E.M.) by the German Federal Environmental Foundation and by the grants of the German Research Foundation contract grant number CRC 1052 "Obesity mechanisms" Project A1 (AV) and WI 3342/3-1 (A.V.W.).

\section{Author details}

${ }^{1}$ Department of Neurology, Max Planck Institute for Human Cognitive and Brain Sciences, Leipzig, Germany. ${ }^{2}$ Berlin School of Mind and Brain, HumboldtUniversität zu Berlin, Berlin, Germany. ${ }^{3}$ Charité-Universitätsmedizin Berlin, Humboldt-Universität zu Berlin, Berlin, Germany. ${ }^{4}$ Helmholtz Centre for Environmental Research GmbH_UFZ, Leipzig, Germany

\section{Authors' contributions}

E.M., A.V. and A.V.W. designed research; E.M. conducted research; E.M., S.H. and A.V.W. analyzed data; E.M. and A.V.W. wrote the paper; E.M., A.V. and A.V.W. had primary responsibility for final content. All authors read and approved the final manuscript.

\section{Conflict of interest}

The authors declare that they have no conflict of interest.

\section{Publisher's note}

Springer Nature remains neutral with regard to jurisdictional claims in published maps and institutional affiliations.
Supplementary Information accompanies this paper at (https://doi.org/ 10.1038/s41398-019-0552-0).

Received: 20 February 2019 Revised: 22 June 2019 Accepted: 17 July 2019 Published online: 12 September 2019

References

1. GOV.UK. National Diet and Nutrition Survey: headline results from years 1, 2 and 3 (combined) of the rolling programme 2008/09-2010/11. https://www gov.uk/government/statistics/national-diet-and-nutrition-survey-headlineresults-from-years-1-2-and-3-combined-of-the-rolling-programme-200809201011 (2012).

2. V. E. B. U. Deutschland \& Joy, S. Anzahl der Veganer und Vegetarier in Deutschland. Stand 31, 2016 (2015).

3. Mensink, G., Barbosa, C. L. \& Brettschneider, A.-K. Verbreitung der vegetarischen Ernährungsweise in Deutschland 1, (2016).

4. The Vegetarian Resource Group. How many adults in the U.S. are vegetarian and vegan? http://www.vrg.org/nutshell/Polls/ 2016_adults_veg.htm (2016).

5. Rosenfeld, D. L. \& Burrow, A. L. Vegetarian on purpose: understanding the motivations of plant-based dieters. Appetite 116, 456-463 (2017).

6. Orlich, M. J. et al. Vegetarian dietary patterns and mortality in Adventist Health Study 2. JAMA Intern. Med. 173, 1230-1238 (2013).

7. Le, L. T. \& Sabaté, J. Beyond meatless, the health effects of vegan diets: findings from the Adventist cohorts. Nutrients 6, 2131-2147 (2014).

8. Mihrshahi, S. et al. Vegetarian diet and all-cause mortality: evidence from a large population-based Australian cohort-the 45 and up study. Prev. Med. 97, 1-7 (2017).

9. Key, T. J. et al. Mortality in British vegetarians: results from the European Prospective Investigation into Cancer and Nutrition (EPIC-Oxford). Am. J. Clin. Nutr. 89, 1613S-1619S (2009).

10. Fung, T. T. et al. Low-carbohydrate diets and all-cause and cause-specific mortalitytwo cohort studies. Ann. Intern. Med. 153, 289-298 (2010).

11. Song, M. et al. Association of animal and plant protein intake with all-cause and cause-specific mortality. JAMA Intern. Med. 176, 1453-1463 (2016).

12. Hu, F. B. Plant-based foods and prevention of cardiovascular disease: an overview. Am. J. Clin. Nutr. 78, 544S-551S (2003).

13. Tonstad, S., Butler, T., Yan, R. \& Fraser, G. E. Type of vegetarian diet, body weight, and prevalence of type 2 diabetes. Diabetes Care $\mathbf{3 2}$, 791-796 (2009).

14. McEvoy, C. T., Temple, N. \& Woodside, J. V. Vegetarian diets, low-meat diets and health: a review. Public Health Nutr. 15, 2287-2294 (2012).

15. Glick-Bauer, M. \& Yeh, M.-C. The health advantage of a vegan diet: exploring the gut microbiota connection. Nutrients 6, 4822-4838 (2014).

16. Appleby, P. N. \& Key, T. J. The long-term health of vegetarians and vegans. Proc. Nutr. Soc. 75, 287-293 (2016).

17. Eichelmann, F., Schwingshackl, L., Fedirko, V. \& Aleksandrova, K. Effect of plant-based diets on obesity-related inflammatory profiles: a systematic review and meta-analysis of intervention trials. Obes. Rev. 17, 1067-1079 (2016).

18. McMacken, M. \& Shah, S. A plant-based diet for the prevention and treatment of type 2 diabetes. J. Geriatr. Cardiol. 14, 342 (2017).

19. Rogers, G. B. et al. From gut dysbiosis to altered brain function and mental illness: mechanisms and pathways. Mol. Psychiatry 21, 738-748 (2016).

20. Hibbeln, J. R., Northstone, K., Evans, J. \& Golding, J. Vegetarian diets and depressive symptoms among men. J. Affect Disord. 225, 13-17 (2018).

21. Forestell, C. A. \& Nezlek, J. B. Vegetarianism, depression, and the five factor model of personality. Ecol. Food Nutr. 57, 246-259 (2018).

22. Matta, J. et al. Depressive symptoms and vegetarian diets: results from the constances cohort. Nutrients 10, 1695 (2018).

23. Agarwal, U. et al. A multicenter randomized controlled trial of a nutrition intervention program in a multiethnic adult population in the corporate setting reduces depression and anxiety and improves quality of life: the GEICO study. Am. J. Health Promot. 29, 245-254 (2015).

24. Beezhold, B., Radnitz, C., Rinne, A. \& DiMatteo, J. Vegans report less stress and anxiety than omnivores. Nutr. Neurosci. 18, 289-296 (2015).

25. Barnard, N. D., Levin, S. M. \& Yokoyama, Y. A systematic review and metaanalysis of changes in body weight in clinical trials of vegetarian diets. $J$. Acad. Nutr. Diet. 115, 954-969 (2015). 
26. Huang, R.-Y., Huang, C.-C., Hu, F. B. \& Chavarro, J. E. Vegetarian diets and weight reduction: a meta-analysis of randomized controlled trials. J. Gen. Intern. Med. 31, 109-116 (2016).

27. Benatar, J. R. \& Stewart, R. A. H. Cardiometabolic risk factors in vegans: a metaanalysis of observational studies. PLOS ONE 13, e0209086 (2018).

28. Lee, Y.-M. et al. Effect of a brown rice based vegan diet and conventiona diabetic diet on glycemic control of patients with type 2 diabetes: a 12-week randomized clinical trial. PLoS ONE 11, e0155918 (2016).

29. Jenkins, D. J. A. et al. Effect of a 6-month vegan low-carbohydrate ('ECoAtkins') diet on cardiovascular risk factors and body weight in hyperlipidaemic adults: a randomised controlled trial. BMJ Open 4, e003505 (2014).

30. Jenkins, D. J. A. et al. The effect of a plant-based low-carbohydrate ("EcoAtkins") diet on body weight and blood lipid concentrations in hyperlipidemic subjects. Arch. Intern. Med. 169, 1046-1054 (2009).

31. Barnard, N. D. et al. A low-fat vegan diet and a conventional diabetes diet in the treatment of type 2 diabetes: a randomized, controlled, 74-wk clinical trial. Am. J. Clin. Nutr. https://doi.org/10.3945/ajen.2009.26736H (2009).

32. Kahleova, H., Hill M. \& Pelikánova, T. Vegetarian vs. conventional diabetic diet -a 1-year follow-up. Cor Vasa 56. https://doi.org/10.1016/j. crvasa.2013.12.004 (2016).

33. Turner-McGrievy, G. M., Davidson, C. R., Wingard, E. E., Wilcox, S. \& Frongillo, E. A. Comparative effectiveness of plant-based diets for weight loss: a randomized controlled trial of five different diets. Nutrition 31, 350-358 (2015).

34. Wing, R. R. \& Phelan, S. Long-term weight loss maintenance. Am. J. Clin. Nutr. 82, 222S-225S (2005).

35. David, L. A. et al. Diet rapidly and reproducibly alters the human gut microbiome. Nature 505, 559-563 (2014).

36. Wu, G. D. et al. Linking long-term dietary patterns with gut microbial enterotypes. Science (80-) 334, 105-108 (2011).

37. Kaartinen, $\mathrm{K}$. et al. Vegan diet alleviates fibromyalgia symptoms. Scand. J. Rheumatol. 29, 308-313 (2000).

38. Yadav, V. et al. Low-fat, plant-based diet in multiple sclerosis: a randomized controlled trial. Mult. Scler. Relat. Disord. 9, 80-90 (2016).

39. Rauma, A. L., Nenonen, M., Helve, T. \& Hänninen, O. Effect of a strict vegan diet on energy and nutrient intakes by Finnish rheumatoid patients. Eur. J. Clin. Nutr. 47, 747-749 (1993).

40. Elkan, A.-C. et al. Gluten-free vegan diet induces decreased LDL and oxidized LDL levels and raised atheroprotective natural antibodies against phosphorylcholine in patients with rheumatoid arthritis: a randomized study. Arthritis Res. Ther. 10, R34 (2008).

41. Karlsson, J. et al. Predictors and effects of long-term dieting on mental wellbeing and weight loss in obese women. Appetite 23, 15-26 (1994).

42. Beezhold, B. L. \& Johnston, C. S. Restriction of meat, fish, and poultry in omnivores improves mood: a pilot randomized controlled trial. Nutr. J. 11, 9 (2012).

43. Yokoyama, Y., Barnard, N. D., Levin, S. M. \& Watanabe, M. Vegetarian diets and glycemic control in diabetes: a systematic review and meta-analysis. Cardiovasc. Diagn. Ther. 4, 373-382 (2014).

44. Sutliffe, J. T., Wilson, L. D., de Heer, H. D., Foster, R. L. \& Carnot, M. J. C-reactive protein response to a vegan lifestyle intervention. Complement Ther. Med. 23, 32-37 (2015).

45. Strasser, B., Gostner, J. M. \& Fuchs, D. Mood, food, and cognition: role of tryptophan and serotonin. Curr. Opin. Clin. Nutr. Metab. Care 19, 55-61 (2016).

46. O'Mahony, S. M., Clarke, G., Borre, Y. E., Dinan, T. G. \& Cryan, J. F. Serotonin, tryptophan metabolism and the brain-gut-microbiome axis. Behav. Brain Res. 277, 32-48 (2015).

47. Davey, G. K. et al. EPIC-Oxford: lifestyle characteristics and nutrient intakes in a cohort of 33883 meat-eaters and 31546 non meat-eaters in the UK. Public Health Nutr. 6, 259-268 (2003).

48. Schüpbach, R., Wegmüller, R., Berguerand, C., Bui, M. \& Herter-Aeberli, I. Micronutrient status and intake in omnivores, vegetarians and vegans in Switzerland. Eur. J. Nutr. 56, 283-293 (2017).

49. Clarys, P. et al. Comparison of nutritional quality of the vegan, vegetarian, semi-vegetarian, pesco-vegetarian and omnivorous diet. Nutrients $\mathbf{6}$, 1318-1332 (2014).

50. Park, J. E., Miller, M., Rhyne, J., Wang, Z. \& Hazen, S. L. Differential effect of short-term popular diets on TMAO and other cardio-metabolic risk markers. Nutr. Metab. Cardiovasc. Dis. 29, 513-517 (2019).

51. Psichas, A. et al. The short chain fatty acid propionate stimulates GLP-1 and PYY secretion via free fatty acid receptor 2 in rodents. Int J. Obes. 39, 424 (2015).
52. Lin, H. V. et al. Butyrate and propionate protect against diet-induced obesity and regulate gut hormones via free fatty acid receptor 3-independent mechanisms. PLOS ONE 7, e35240 (2012).

53. Canfora, E. E., Jocken, J. W. \& Blaak, E. E. Short-chain fatty acids in control of body weight and insulin sensitivity. Nat. Rev. Endocrinol. 11, 577 (2015).

54. Guo, Y. et al. Physiological evidence for the involvement of peptide $Y Y$ in the regulation of energy homeostasis in humans. Obesity 14, 1562-1570 (2006).

55. Holzer, P., Reichmann, F. \& Farzi, A. Neuropeptide Y, peptide $Y$ Y and pancreatic polypeptide in the gut-brain axis. Neuropeptides 46, 261-274 (2012).

56. Kendall, C. W. C., Esfahani, A. \& Jenkins, D. J. A. The link between dietary fibre and human health. Food Hydrocoll. 24, 42-48 (2010).

57. Reynolds, A. et al. Carbohydrate quality and human health: a series of systematic reviews and meta-analyses. Lancet. https://doi.org/10.1016/S01406736(18)31809-9 (2019).

58. Menni, C. et al. Gut microbiome diversity and high-fibre intake are related to lower long-term weight gain. Int. J. Obes. 41, 1099 (2017).

59. Van Gaal, L. F., Mertens, I. L. \& Christophe, E. Mechanisms linking obesity with cardiovascular disease. Nature 444, 875 (2006).

60. Ferreira, C. M. et al. The central role of the gut microbiota in chronic inflammatory diseases. J. Immunol. Res. 2014, https:/doi.org/10.1155/2014/ 689492 (2014)

61. Wersching, $H$. et al. Serum C-reactive protein is linked to cerebral microstructural integrity and cognitive function. Neurology 74, 1022-1029 (2010).

62. Gu, Y. et al. Circulating inflammatory biomarkers in relation to brain structural measurements in a non-demented elderly population. Brain Behav. Immun. 65, 150-160 (2017).

63. Lampe, L. et al. Visceral obesity relates to deep white matter hyperintensities via inflammation. Ann. Neurol. 85, 194-203 (2018).

64. Schmidt, R. et al. Early inflammation and dementia: a 25-year follow-up of the Honolulu-Asia Aging Study. Ann. Neurol. 52, 168-174 (2002).

65. Rosano, C., Marsland, A. L. \& Gianaros, P. J. Maintaining brain health by monitoring inflammatory processes: a mechanism to promote successfu aging. Aging Dis. 3, 16 (2012)

66. Tangney, C. C. et al. Relation of DASH-and Mediterranean-like dietary patterns to cognitive decline in older persons. Neurology 83, 1410-1416 (2014).

67. Craddock, J. C., Probst, Y. \& Peoples, G. Vegetarian nutrition-comparing physical performance of omnivorous and vegetarian athletes. J. Nutr. Intermed. Metab. 4, 19 (2016).

68. Liu, R. H. Health benefits of fruit and vegetables are from additive and synergistic combinations of phytochemicals. Am. J. Clin. Nutr. 78, 517S-520S (2003).

69. Boffetta, P. et al. Fruit and vegetable intake and overall cancer risk in the European Prospective Investigation into Cancer and Nutrition (EPIC). J. Natl. Cancer Inst. 102, 529-537 (2010).

70. Reczek, C. R. \& Chandel, N. S. Revisiting vitamin C and cancer. Science (80-) 350, 1317-1318 (2015)

71. Probst, Y. C., Guan, V. X. \& Kent, K. Dietary phytochemical intake from foods and health outcomes: a systematic review protocol and preliminary scoping. BMJ Open 7, e013337 (2017).

72. Hartmann, R. \& Meisel, H. Food-derived peptides with biological activity: from research to food applications. Curr. Opin. Biotechnol. 18, 163-169 (2007).

73. Tillisch, $\mathrm{K}$. et al. Consumption of fermented milk product with probiotic modulates brain activity. Gastroenterology 144, 1394-1401 (2013).

74. Gibson, G. R. et al. Expert consensus document: The International Scientific Association for Probiotics and Prebiotics (ISAPP) consensus statement on thedefinition and scope of prebiotics. Nat. Rev. Gastroenterol. Hepatol. 14, 491 (2017).

75. Nisha, A. R. Antibiotic residues-a global health hazard. Vet. World 1, 375-377 (2008).

76. Wang, $\mathrm{H}$. et al. Antibiotic residues in meat, milk and aquatic products in Shanghai and human exposure assessment. Food Control 80, 217-225 (2017).

77. Bertazzi, P. A. et al. Health effects of dioxin exposure: a 20-year mortality study. Am. J. Epidemiol. 153, 1031-1044 (2001).

78. Bouvard, V. et al. Carcinogenicity of consumption of red and processed meat. Lancet Oncol. 16, 1599-1600 (2015).

79. Van Audenhaege, M. et al. Impact of food consumption habits on the pesticide dietary intake: comparison between a French vegetarian and the general population. Food Addit. Contam. 26, 1372-1388 (2009).

80. Jacobs, D. R. \& Tapsell, L. C. Food synergy: the key to a healthy diet. Proc. Nutr. Soc. 72, 200-206 (2013). 
81. Kawano, Y. \& Yanai, K. Foodcam: a real-time food recognition system on a smartphone. Multimed. Tools Appl. 74, 5263-5287 (2015).

82. Garcia-Perez, I. et al. Objective assessment of dietary patterns by use of metabolic phenotyping: a randomised, controlled, crossover trial. Lancet Diabetes Endocrinol. 5, 184-195 (2017).

83. Turner-McGrievy, G. M. et al. Changes in nutrient intake and dietary quality among participants with type 2 diabetes following a low-fat vegan diet or a conventional diabetes diet for 22 weeks. J. Am. Diet. Assoc. 108, 1636-1645 (2008).

84. Gilsing, A. M. J. et al. Serum concentrations of vitamin B12 and folate in British male omnivores, vegetarians and vegans: results from a cross-sectional analysis of the EPIC-Oxford cohort study. Eur. J. Clin. Nutr. 64, 933-939 (2010).

85. Allen, L. H. How common is vitamin B-12 deficiency? Am. J. Clin. Nutr. 89, 693S-696S (2008).

86. Pawlak, R., Parrott, S. J., Raj, S., Cullum-Dugan, D. \& Lucus, D. How prevalent is vitamin B12 deficiency among vegetarians? Nutr. Rev. 71, 110-117 (2013).

87. Rizzo, G. et al. Vitamin B12 among vegetarians: status, assessment and supplementation. Nutrients $\mathbf{8}, 767$ (2016).

88. Stabler, S. P. Vitamin B12 deficiency. N. Engl. J. Med. 368, 149-160 (2013).

89. Köbe, T. et al. Vitamin B-12 concentration, memory performance, and hippocampal structure in patients with mild cognitive impairment, 2. Am. J. Clin. Nutr. 103, 1045-1054 (2016).

90. Ganguly, P. \& Alam, S. F. Role of homocysteine in the development of cardiovascular disease. Nutr. J. 14, 6 (2015).

91. McCaddon, A., Regland, B., Hudson, P. \& Davies, G. Functional vitamin B12 deficiency and Alzheimer disease. Neurology 58, 1395-1399 (2002).

92. Moore, E. et al. Cognitive impairment and vitamin B12: a review. Int. Psychogeriatr. 24, 541-556 (2012).

93. Spence, J. D. Metabolic vitamin B12 deficiency: a missed opportunity to prevent dementia and stroke. Nutr. Res. 36, 109-116 (2016).

94. Nexo, E. \& Hoffmann-Lücke, E. Holotranscobalamin, a marker of vitamin B-12 status: analytical aspects and clinical utility. Am. J. Clin. Nutr. 94, 359S-365S (2011).

95. Haider, L. M., Schwingshackl, L., Hoffmann, G. \& Ekmekcioglu, C. The effect of vegetarian diets on iron status in adults: a systematic review and metaanalysis. Crit. Rev. Food Sci. Nutr. 58, 1359-1374 (2018).

96. Lozoff, B. \& Georgieff, M. K. et al. Iron deficiency and brain development. Semin. Pediatr. Neurol. 13, 158-165 (2006).

97. Ayton, S. et al. Brain iron is associated with accelerated cognitive decline in people with Alzheimer pathology. Mol. Psychiatry 1, https://doi.org/10.1038/ s41380-019-0375-7 (2019).

98. Murray-Kolb, L. E. \& Beard, J. L. Iron treatment normalizes cognitive functioning in young women. Am. J. Clin. Nutr. 85, 778-787 (2007).

99. Beard, J. Iron deficiency alters brain development and functioning. J. Nutr. 133, 1468S-1472S (2003)

100. Melina, V., Craig, W. \& Levin, S. Position of the Academy of Nutrition and Dietetics: vegetarian diets. J. Acad. Nutr. Diet. 116, 1970-1980 (2016).

101. Richter, M. et al. For the German Nutrition Society (DGE)(2016) Vegan diet Position of the German Nutrition Society (DGE). Ernaehrungsumschau 63, 92-102 (2016)

102. Peterson, J. et al. The NIH human microbiome project. Genome Res. 19, 2317-2323 (2009).

103. Arumugam, M. et al. Enterotypes of the human gut microbiome. Nature $\mathbf{4 7 3}$ 174-180 (2013).

104. Bamberger, C. et al. A walnut-enriched diet affects gut microbiome in healthy Caucasian subjects: a randomized, controlled trial. Nutrients 10, 244 (2018).

105. Holscher, H. D. et al. Walnut consumption alters the gastrointestinal microbiota, microbially derived secondary bile acids, and health markers in healthy adults: a randomized controlled trial. J. Nutr. 148, 861-867 (2018).

106. Hjorth, M. F. et al. Pre-treatment microbial Prevotella-to-Bacteroides ratio, determines body fat loss success during a 6-month randomized controlled diet intervention. Int J. Obes. 42, 580 (2018).

107. Hansen, T. H. et al. Impact of a vegan diet on the human salivary microbiota. Sci. Rep. 8, 5847 (2018).

108. Kim, M., Hwang, S., Park, E. \& Bae, J. Strict vegetarian diet improves the risk factors associated with metabolic diseases by modulating gut microbiota and reducing intestinal inflammation. Environ. Microbiol. Rep. 5, 765-775 (2013).

109. Zimmer, J. et al. A vegan or vegetarian diet substantially alters the human colonic faecal microbiota. Eur. J. Clin. Nutr. 66, 53-60 (2012).
110. De Filippis, F., Pellegrini, N., Laghi, L., Gobbetti, M. \& Ercolini, D. Unusual subgenus associations of faecal Prevotella and Bacteroides with specific dietary patterns. Microbiome 4, 57 (2016).

111. Kumar, M., Babaei, P., Ji, B. \& Nielsen, J. Human gut microbiota and healthy aging: Recent developments and future prospective. Nutr. Health Aging $\mathbf{4}$, 3-16 (2016).

112. Wu, G. D. et al. Comparative metabolomics in vegans and omnivores reveal constraints on diet-dependent gut microbiota metabolite production. Gut 65, 63-72 (2014).

113. Morrison, D. J. \& Preston, T. Formation of short chain fatty acids by the gut microbiota and their impact on human metabolism. Gut Microbes 7, 189-200 (2016).

114. Wanders, A. J. et al. Effects of dietary fibre on subjective appetite, energy intake and body weight: a systematic review of randomized controlled trials. Obes. Rev. 12, 724-739 (2011).

115. Brunkwall, L. \& Orho-Melander, M. The gut microbiome as a target for prevention and treatment of hyperglycaemia in type 2 diabetes: from current human evidence to future possibilities. Diabetologia 60, 943-951 (2017).

116. Lach, G., Schellekens, H., Dinan, T. G. \& Cryan, J. F. Anxiety, depression, and the microbiome: a role for gut peptides. Neurotherapeutics 15, 36-59 (2018).

117. Bagga, D. et al. Influence of 4-week multi-strain probiotic administration on resting-state functional connectivity in healthy volunteers. Eur. J. Nutr. 58, 1821-1827 (2018).

118. Foster, J. A. \& Neufeld, K.-A. M. Gut-brain axis: how the microbiome influences anxiety and depression. Trends Neurosci. 36, 305-312 (2013).

119. Saulnier, D. M. et al. The intestinal microbiome, probiotics and prebiotics in neurogastroenterology. Gut Microbes 4, 17-27 (2013).

120. Maes, M., Kubera, M., Leunis, J.-C. \& Berk, M. Increased IgA and IgM responses against gut commensals in chronic depression: further evidence for increased bacterial translocation or leaky gut. J. Affect Disord. 141, 55-62 (2012).

121. Huang, R., Wang, K. \& Hu, J. Effect of probiotics on depression: a systematic review and meta-analysis of randomized controlled trials. Nutrients $\mathbf{8}, 483$ (2016).

122. Heintz-Buschart, A. et al. Integrated multi-omics of the human gut microbiome in a case study of familial type 1 diabetes. Nat. Microbiol. 2, 16180 (2016).

123. Strang, S. et al. Impact of nutrition on social decision making. Proc. Natl Acad. Sci. 114, 6510-6514 (2017)

124. Osadchiy, V. et al. Correlation of tryptophan metabolites with connectivity of extended central reward network in healthy subjects. PLOS ONE 13, e0201772 (2018).

125. Franzosa, E. A. et al. Sequencing and beyond: integrating molecular'omics' for microbial community profiling. Nat. Rev. Microbiol. 13, 360-372 (2015).

126. Fernandez-Real, J.-M. et al. Gut microbiota interacts with brain microstructure and function. J. Clin. Endocrinol. Metab. 100, 4505-4513 (2015).

127. Allen, A. P. et al. Bifidobacterium longum 1714 as a translational psychobiotic: modulation of stress, electrophysiology and neurocognition in healthy volunteers. Transl. Psychiatry 6, e939 (2016).

128. Harach, T. et al. Reduction of Abeta amyloid pathology in APPPS1 transgenic mice in the absence of gut microbiota. Sci. Rep. 7, 41802 (2017).

129. Huhn, S., Masouleh, S. K., Stumvoll, M., Villringer, A. \& Witte, A. V. Components of a Mediterranean diet and their impact on cognitive functions in aging. Front Aging Neurosci 7, 132 (2015).

130. Larsson, S. C., Wallin, A. \& Wolk, A. Dietary approaches to stop hypertension diet and incidence of stroke: results from 2 prospective cohorts. Stroke 47, 986-990 (2016).

131. van de Rest, $\mathrm{O}$. et al. Effect of fish oil on cognitive performance in older subjects: a randomized, controlled trial. Neurology 71, 430-438 (2008).

132. Witte, A. V. et al. Long-chain omega-3 fatty acids improve brain function and structure in older adults. Cereb. Cortex 24, 3059-3068 (2013).

133. Witte, A. V., Kerti, L., Margulies, D. S. \& Flöel, A. Effects of resveratrol on memory performance, hippocampal functional connectivity, and glucose metabolism in healthy older adults. J. Neurosci. 34, 7862-7870 (2014).

134. Brickman, A. M. et al. Enhancing dentate gyrus function with dietary flavanols improves cognition in older adults. Nat. Neurosci. 17, 1798 (2014).

135. Martínez-González, M. A. et al. Benefits of the Mediterranean diet: insights from the PREDIMED study. Prog. Cardiovasc. Dis. 58, 50-60 (2015).

136. Huhn, S. et al. Effects of resveratrol on memory performance, hippocampus connectivity and microstructure in older adults — a randomized controlled trial. Neuroimage (2018). 
137. Rosenberg, A. et al. Multidomain lifestyle intervention benefits a large elderly population at risk for cognitive decline and dementia regardless of baseline characteristics: The FINGER trial. Alzheimer's. Dement. 14, 263-270 (2018).

138. Turnbaugh, P. J. Microbes and diet-induced obesity: fast, cheap, and out of control. Cell Host Microbe 21, 278-281 (2017)

139. Turner-Mc Grievy, G. M., Barnard, N. D. \& Scialli, A. R. A two-year randomized weight loss trial comparing a vegan diet to a more moderate low-fat diet* Obesity 15, 2276-2281 (2007).

140. Burke, L. E. et al. A randomized clinical trial of a standard versus vegetarian diet for weight loss: the impact of treatment preference. Int. J. Obes. 32, 166-176 (2008).

141. Barnard, N. D. et al. A low-fat vegan diet and a conventional diabetes diet in the treatment of type 2 diabetes: a randomized, controlled, 74-wk clinical trial. Am. J. Clin. Nutr. 89, 1588S-1596S (2009).

142. Marniemi, J., Seppänen, A. \& Hakala, P. Long-term effects on lipid metabolism of weight reduction on lactovegetarian and mixed diet. Int. J. Obes. 14, 113-125 (1990)

143. Acharya, S. D., Brooks, M. M., Evans, R. W., Linkov, F. \& Burke, L. E. Weight loss is more important than the diet type in improving adiponectin levels among overweight/obese adults. J. Am. Coll. Nutr. 32, 264-271 (2013).

144. Wright, N., Wilson, L., Smith, M., Duncan, B. \& McHugh, P. The BROAD study: A randomised controlled trial using a whole food plant-based diet in the community for obesity, ischaemic heart disease or diabetes. Nutr. Diabetes $\mathbf{7}$, e256 (2017).

145. Turner-McGrievy, G. M., Davidson, C. R., Wingard, E. E. \& Billings, D. L. Low glycemic index vegan or low-calorie weight loss diets for women with polycystic ovary syndrome: a randomized controlled feasibility study. Nutr. Res. 34, 552-558 (2014)

146. Kahleova, H. et al. Vegetarian diet improves insulin resistance and oxidative stress markers more than conventional diet in subjects with Type 2 diabetes. Diabet. Med 28, 549-559 (2011).

147. Ferdowsian, H. R. et al. A multicomponent intervention reduces body weight and cardiovascular risk at a GEICO corporate site. Am. J. Heal. Promot 24, 384-387 (2010).

148. Mishra, S. et al. A multicenter randomized controlled trial of a plantbased nutrition program to reduce body weight and cardiovascular risk in the corporate setting: the GEICO study. Eur. J. Clin. Nutr. 67, 718 (2013).

149. Agarwal, U. et al. A multicenter randomized controlled trial of a nutrition intervention program in a multiethnic adult population in the corporate setting reduces depression and anxiety and improves quality of life: the GEICO study. Am. J. Heal. Promot 29, 245-254 (2015).

150. Kahleova, H., Dort, S., Holubkov, R. \& Barnard, N. A plant-based high-carbohydrate, low-fat diet in overweight individuals in a 16-week randomized clinical trial: the role of carbohydrates. Nutrients 10, 1302 (2018).

151. Barnard, N., Scialli, A. R., Bertron, P., Hurlock, D. \& Edmonds, K. Acceptability of a therapeutic low-fat, vegan diet in premenopausal women. J. Nutr. Educ. 32, 314-319 (2000).

152. Gardner, C. D. et al. The effect of a plant-based diet on plasma lipids in hypercholesterolemic adults: a randomized trial. Ann. Intern. Med. 142, 733 (2005).

153. Macknin, M. et al. Plant-based, no-added-fat or American Heart Association diets: impact on cardiovascular risk in obese children with hypercholesterolemia and their parents. J. Pediatr. 166, 953-959 (2015).

154. Sciarrone, S. E. et al. Biochemical and neurohormonal responses to the introduction of a lacto-ovovegetarian diet. J. Hypertens. 11, 849-860 (1993).

155. Alleman, R. J., Harvey, I. C., Farney, T. M. \& Bloomer, R. J. Both a traditional and modified Daniel Fast improve the cardio-metabolic profile in men and women. Lipids Health Dis. 12, 114 (2013).

156. Neacsu, M., Fyfe, C., Horgan, G. \& Johnstone, A. M. Appetite control and biomarkers of satiety with vegetarian (soy) and meat-based high-protein diets for weight loss in obese men: a randomized crossover trial-. Am. J. Clin Nutr. 100, 548-558 (2014).

157. Koebnick, C. et al. Double-blind, randomized feedback control fails to improve the hypocholesterolemic effect of a plant-based low-fat diet in patients with moderately elevated total cholesterol levels. Eur. J. Clin. Nutr. 58, 1402 (2004).

158. Kjeldsen-Kragh, J., Haugen, M., Førre, Ø., Laache, H. \& Malt, U. F. Vegetarian diet for patients with rheumatoid arthritis: can the clinical effects be explained by the psychological characteristics of the patients? Rheumatology 33, 569-575 (1994).

159. Bunner, A. E., Agarwal, U., Gonzales, J. F., Valente, F. \& Barnard, N. D. Nutrition intervention for migraine: a randomized crossover trial. J. Headache Pain. 15, 69 (2014).

160. Kahleova, H., Hrachovinova, T., Hill, M. \& Pelikanova, T. Vegetarian diet in type 2 diabetes-improvement in quality of life, mood and eating behaviour. Diabet. Med 30, 127-129 (2013).

161. Turner-McGrievy, G. M. et al. Randomization to plant-based dietary approaches leads to larger short-term improvements in Dietary Inflammatory Index scores and macronutrient intake compared with diets that contain meat. Nutr. Res. 35, 97-106 (2015) 